\title{
Grand challenges in alloimmunity and transplantation
}

\author{
Antoine Toubert* \\ Université Paris Diderot, Sorbonne Paris Cité, Institut Universitaire d'Hématologie, INSERM UMR940, Laboratoire d'Immunologie et d'Histocompatibilité, Laboratoire \\ Jean Dausset, Hôpital Saint-Louis, Assistance Publique-Hôpitaux de Paris, Paris, France \\ *Correspondence: antoine.toubert@univ-paris-diderot.fr
}

\section{THE PIONEERING TIMES}

Although the concept of Transplantation could be traced back as early as Saint Cosmas and Damian most famous miracle, grafting of a leg to replace a patient's ulcered leg, it is not before the 50's that it entered reality. Important steps were made before, from sewing vessels to understanding the basis of the major histocompatibility complex (MHC) in mice (Snell, 1986). This medical field was pioneered in renal transplantation with nearly concomitant attempts in Paris, London, Edinburgh, Boston. The first successful attempt was carried out in Boston by Joseph Murray in 1954. The donor and recipient were identical twins, showing indeed the importance of Genetic compatibility. Organ transplantation (OT) then became one of the most exciting "success story" of modern Medicine, admixing advances in Surgery and intensive care Medicine, Immunology, Genetics, and Pharmacology. The crucial point was first to avoid acute graft rejection which quickly appeared to be immune-mediated. The development of potent immunosuppressive (IS) drugs (corticosteroids, calcineurin inhibitors) opened the way to clinical transplantation. Most critical landmarks were in 1957 the first hepatic transplantation followed by transplantation of other organs, in 1967 the first successful heart transplantation, lung (1968), trachea (1979), pancreas, and more recently hands (2000) and facial tissue (2005). In hematopoietic stem-cell transplantation (HSCT), the initial report of the use of bone marrow transplantation in cancer treatment was made by Thomas in 1957 . Several Nobel prizes were awarded to some of the transplantation "founding fathers": Medawar and Burnet (1960), Benacerraf, Dausset and Snell (1980), Murray and Thomas (1990).

\section{TRANSPLANTATION MEDICINE AND THE GOLDEN AGE OF HLA}

It is a remarkable that HLA, the human MHC, discovery was due to a deliberate medical action, transplantation, and not to a naturally occurring disease. Actually, if HLA governs the allogeneic response, it is obviously not its primary function. HLA, discovered in the 50's in Dausset, van Rood, and Mc Devitt laboratories, among others, appeared from the 70's onward as a central actor of the immune response. MHC molecules bind peptides from antigens (of viral, tumoral, or "self" origin) to be presented to the T-cell antigen receptor. This has been definitely evidenced by several crystallographic structures of MHC-peptide complexes, the first being the HLA-A2 molecule binding endogenous peptides by Bjorkman in the Wiley's laboratory. The role of HLA is therefore to bind peptides from foreign proteins and then be recognized, as a combination, by $\mathrm{T}$ cells that ensure the elimination of the source of peptide. It appears clearly how this system could be efficient to recognize self from "allogeneic" non-self, i.e., cells or tissues from another individual of the same species but with a different genetic background and a different array of HLApeptide complexes. Indeed, the frequency of alloreactive naïve $\mathrm{T}$ cells is about 100 to 1000-fold higher than that of naïve conventional $\mathrm{T}$ cells. This dramatically efficient system has to be kept under control to avoid graft rejection. In HSCT, a situation where a lymphopenic host receives an appropriate conditioning regimen (total body irradiation, chemotherapy) to accept the allogeneic source of cells, the risk is to develop a potentially fatal "graft-versus-host" (GVH) reaction, based on the same concept of allogeneic reactivity but directed toward the recipient self antigens. Considerable advances have been made following these basic discoveries:

A In histocompatibility and immunogenetics, leading to the definition of more than 4000 HLA alleles. Technically, HLA typing evolved from serology to Molecular Biology including now high speed sequencing. In the meantime, besides classical HLA loci (HLA-A, -B, $-C$, -DR, -DP, DQ), others were descri- bed in the HLA locus (HLA-G, -E, MICA, MICB) raising the issue of their role in Transplantation Immunology.

A In Pharmacology, with the development of new IS drugs (mycophenolate mofetil, mTOR inhibitors) and Biotherapies: rabbit antithymocyte globulin, monoclonal antibodies directed toward the interleukin-2 receptor alpha-chain (daclizumab and basiliximab) or specific for CD52 (alemtuzumab).

A In the clinical management of the patients and of the drugs with sometimes severe adverse events, especially infections and cancer.

All these progresses combined explain the development of this highly specialized medical field, giving hope to many patients affected by an end-stage organ failure or a fatal hematological malignancy.

\section{CHALLENGES IN TRANSPLANTATION IMMUNOLOGY AND MEDICINE: "INCREASE, IMPROVE, INNOVATE"}

These few preliminary remarks show that from the very beginning, Transplantation was a remarkable integration of Medicine and basic Immunology, Pharmacology, Epidemiology, and Ethics, to name only the most relevant fields. In many ways, Transplantation was a leading force of innovation in unrelated medical specialties and vice versa. Which are some of the main limitations we are facing today?

\section{INCREASE TRANSPLANT EFFICIENCY AND DONOR ALLOCATION}

Around 300,000 Europeans are living with a transplanted organ in 2010. Number of transplants in Western countries showed a $45 \%$ increase since 2000. In parallel, there is an increasing shortage of organ donors and thousands are on waiting lists to get transplanted. This foster access to living related donors and leads to allow more frequently organs with a "border line" functionality. In the same time, recipients may already 
have received a successful transplant and need to be re-transplanted. Their age tend to increase, as well as other risk factors common to Western aging populations (obesity, infections). In total, this may impair the future success rate of OT and pushes to improve the definition of genetic matching for an optimal organ allocation. Pretransplant status of the recipient in terms of HLA pre-sensitization, HLA matching or "authorized" mismatching based on scientific algorithms (Duquesnoy, 2011), data analysis from broad sequencing to define more relevant genetic polymorphisms (Pharmacogenetics, non-classical HLA polymorphisms, cytokine, and chemokine gene polymorphisms) are some of the tasks in OT and HSCT as well (Dickinson, 2008). In HSCT, the issue is also to optimize donor allocation and to further develop alternative sources of stem cells already clinically validated (hematopoietic stem cells from cord blood, MHC Haploidentical sibling donor) combined with changes in the procedures of conditioning (non-myeloablative versus myeloablative). This, of course, will require large-scale evaluations in terms of clinical benefit, in the short (acute GVH disease, leukemic relapse) and in the long-term (immune recovery, chronic GVH).

\section{IMPROVE LONG-TERM SURVIVAL OF TRANSPLANTED PATIENTS}

After the successful step of early graft acceptance due to heavy IS, the main issue is now more to improve the long-term survival of solid-organ transplanted patients. Maintenance of IS therapy, especially calcineurin inhibitors, is associated with significant risks of cardiovascular disease, metabolic syndrome, bone loss, opportunistic infections, and an increased rate of malignancies. Similar issues apply to late side effects in allo-HSCT patients (Tichelli et al., 2009). This would require:

^ A better understanding of Tolerance in this clinical setting and especially of so-called "operational tolerance." Tolerance is the focus of the Frontiers Specialty journal "Immunological Tolerance," showing how much the field evolved in the past 10 years. Operational Tolerance, which is "Holy Grail" of transplant immunologists, refers to a situation of long-term acceptance of transplanted organs without requi- rement of indefinite IS. This concept has been documented in renal transplantation in rare cases where patients discontinued their treatment (Saggo et al., 2010). It is more common in liver Transplantation (Levitsky, 2011) which may provide a good "proof of concept" of it. However, there is yet no consensus on when and how IS therapy could be resumed. This highlights the necessity to define clinically applicable biomarkers of operational tolerance and moreover tolerogenic IS conditions. In that view, conceptual advances in the regulatory networks of the expanding list of CD4 T-cell subsets: "natural" regulatory T cells or Treg but also Th17, Th22, Th10. Technological advances (multiparametric flow cytometry and cell-sorting, transcriptomic, and other "-omics" signatures) applied to transplanted patient's follow-up will be of great value, in OT (Brouard et al., 2011) and HSCT as well.

^ Understanding the risk of emergence of malignancies in transplanted patients. In OT, cutaneous cancers but also post-transplant lymphoproliferative diseases (PTLD) are frequently associated with heavy IS and in most cases virally induced. A better knowledge of the immune status of the patients could help to predict cancer occurrence and to personalize the IS regimen. For instance, some IS drugs (such as mTOR inhibitors) seem to carry a lower risk of secondary malignancy. Same concepts could apply to other late side effects, for instance cardiovascular or metabolic disorders, in OT and HSCT as well.

^ In allo-HSCT, improving donor allocation by alternative donor sources is already in the Clinics (Haplo-HSCT, double cord blood transplantation). It gives a chance to patients lacking a suitable donor from unrelated donors registries. Indeed, it is now commonly accepted that cord blood transplantation could be less stringent in terms of HLA matching. However, this should be evaluated further at an allelic HLA typing level. It will also require more tailored prospective viral and immune monitoring strategies according to the setting of the transplant (source of stem cells, conditioning, T-cell deple- tion...), the recipient pre-transplant immune status such as herpes viruses positivity (CMV or EBV) or thymic function and post-transplant events. Besides, relapse remains a major cause of failure in allo-HSCT. This explains the attempts to boost anti-leukemic immune responses by vaccination or cellular immunotherapy.

\section{INNOVATE: TRANSPLANTATION AS A PLATFORM FOR IMMUNOTHERAPY, GENE THERAPY, AND REGENERATIVE MEDICINE}

The paradigm of allogeneic responses and tolerance acquisition and maintenance is getting a new perspective since the development of stem-cell-based regenerative Medicine. Different aspects should be taken into consideration:

^ Cell-based Immunotherapies to induce Tolerance and improve transplant success:

$>$ Use of regulatory $\mathrm{T}$ cells (Tregs), socalled "regulatory" dendritic cells or mesenchymal stem cells endowed with immunosuppressive properties. These approaches are already entering into the Clinics. Extending their use will need to find to a proper balance between "beneficial" and "detrimental" immunosuppression, in other words to derive alloantigenspecific suppression keeping intact the response to third-party pathogen and tumor antigens.

$>$ Improve immune reconstitution and especially thymic function regeneration in allo-HSCT to generate a pool of naïve competent $\mathrm{T}$ cells (Krenger et al., 2011).

$>$ To improve transplant success by adoptive cellular Therapy:

$\checkmark$ With viral or tumor-specific-specific donor derived or allogeneic third-party T cells.

$\checkmark$ With NK cells, based on the KIR/ ligand mismatch concept (Velardi, 2008), to prevent or cure leukemic relapse. Some successful attempts pave the way to large-scale multicentric clinical trials.

ᄉ Genetic engineering in Transplantation:

In allo-HSCT for hematological malignancies, gene transfer approaches could be a powerful way to selectively modulate patient-specific alloreac- 
tivity and anti-tumor responses and prevent or control GVHD. Suicide gene therapy is to date the most advanced approach already implemented in phase I-II clinical trials (Ludo-Stanghellini et al., 2010).

$>$ In OT, xenotransplantation could be an attractive way to circumvent donor availability (Le Bas-Bernardet et al., 2008). Genetic engineering of the donor aims at overcoming xenogeneic barriers by inactivating species-specific genes (such as pig alpha1,3-galactosyltransferase) to reduce complement activation and humoral acute immune responses. Finally, cell-mediated rejection would require similar approaches as above to be kept under control.

^ Regenerative medicine and allogenicity: human pluripotent stem cells (PSCs) include embryonic stem cells (ESCs) and induced PSCs. They are potentially an unlimited source of cells for regenerative medicine, i.e., to repair, restore, maintain or enhance organ function. ESCs express reduced levels of MHC class I molecules and nonMHC class II or costimulatory molecules and could be "immune -privileged." Actually, beyond major obstacles in Safety (tumorigenicity, genetic stability, cell differentiation) and Ethics, their allogenicity, especially in terminally differentiated cells and tissues, will remains a serious concern (Charron et al., 2009).

In summary, advancing such challenges could improve access to transplantation and the quality of life of transplanted patients in the long-term. Moreover, besides the topics discussed above, transplantation is also tightly connected to Ethics, Social Sciences, and Health Economics which are crucial to provide an equal access to transplantation in minorities and developing countries.

\section{REFERENCES}

Brouard, S., Giral, M., Solulillou, J.P., and Ashton-Chess, J. (2011). Elaboration of gene expression-based clinical decision aids for kidney transplantation: where do we stand? Transplantation 91, 691-696.

Charron, D., Suberbielle-Boissel, C., and Al-Daccak, R. (2009). Immunogenicity and allogenicity: a challenge of stem cell therapy. J. Cardiovasc. Transl Res. $2,130-138$.

Dickinson, A. M. (2008). Polymorphisms of cytokine and innate immunity genes and GVHD. Best Pract. Res. Clin. Haematol. 21, 149-164.

Duquesnoy, R. G. (2011). Antibody reactive epitope determination with HLAMatchmaker and its clinical applications. Tissue Antigens 77, 525-534.

Krenger, W., Blazar, B. R., and Hollander, G. A. (2011). Thymic T-cell development in allogeneic stem cell transplantation. Blood 117, 6768-6776.

Le Bas-Bernardet, S., Anegon, I., and Blancho, G. (2008). Progress and prospects: genetic engineering in xenotransplantation. Gene Ther. 15, 1247-1256.

Levitsky,J. (2011). Operational tolerance: past lessons and future prospects. Liver Transpl. 17, 222-232.
Ludo-Stanghellini, M. T., Provasi, E., Bondanza, A., Ciceri, F., Bordignon, C., and Bonini, C. (2010) Clinical impact of suicide gene therapy in allogeneic hematopoietic stem cell transplantation. Hum. Gene Ther. 21, 241-250.

Saggo, P., Perucha, E., Sawitzki, B., Tomiuk, S., Stephens, D. A., Miqueu, P., Chapman, S., Carciun, L., Sergeant, R., Brouard, S., Rovis, F., Jimenez, E., Ballow, A., Giral, M., Rebollo-Mesa, L., Le Moine, A., Braudeau, C., Hilton, R., Gerstmayer, B., Bourcier, K., Sharif, A., Krajewska, M., Lord, G. M., Roberts, L., Goldman, M., Wood, K. J., Newell, K., Seyfert-Margolis, V., Warrens, A. N., Janssen, U., Volk, H. D., Soulilou, J. P., Hernandez-Fuentes, M.P., and Lechler, R. L. (2010). Development of a cross-platform biomarker signature to detect renal transplant tolerance in humans. J. Clin. Invest. 120, 1848-1861.

Snell, G. D. (1986). Some recollections of Peter Gorer and his work on this fiftieth anniversary of his discovery of H-2. Immunogenetics 24, 339-340.

Tichelli, A., Rovo, A., Passweg, J., Schwarze, C. P., Van Lint, M. T., Arat, M., and Socié, G. (2009). Late complications after hematopoietic stem cell transplantation. Expert Rev. Hematol. 5, 583-601.

Velardi,A. (2008). Role of KIRs and KIR ligands in hematopoietic transplantation. Curr. Opin. Immunol. 20, 581-587.

Received: 16 August 2011; accepted: 16 August 2011; published online: 29 August 2011.

Citation: Toubert A (2011) Grand challenges in alloimmunity and transplantation. Front. Immun. 2:38. doi: 10.3389/ fimmu. 2011.00038

This article was submitted to Frontiers in Alloimmunity and Transplantation, a specialty of Frontiers in Immunology. Copyright () 2011 Toubert. This is an open-access article subject to a non-exclusive license between the authors and Frontiers Media SA, which permits use, distribution and reproduction in other forums, provided the original authors and source are credited and other Frontiers conditions are complied with. 